\title{
Numerical Investigation of Endothermic/Exothermic Reaction in MHD Natural Convective Nano-Fluid Flow over a Vertical Cone with Heat Source/Sink
}

\author{
Silpi HAZARIKA and Sahin AHMED* \\ Department of Mathematics, Rajiv Gandhi University, Arunachal Pradesh, India
}

('Corresponding author's e-mail: nanofluid.sahin@gmail.com)

Received: 21 March 2021, Revised: 9 May 2021, Accepted: 16 May 2021

\begin{abstract}
The significance of natural convective MHD flow of nano-fluid over a vertical cone with Brownian motion, viscous dissipation, heat generation, thermophoresis, and chemical reaction in porous surroundings is discussed in this article. The leading non-linear PDEs are transmuted by using applicable similarity transform and the reformed equations, along with the boundary conditions, are numerically solved by finite difference technique of bvp4c code via MATLAB code. The significance of the controlling parameters on velocity, temperature, and concentration are portrayed vividly. Furthermore, skin friction, Nusselt number, and Sherwood number are charted for diverse parameters. It is worth mentioning that impact of heat source $(Q)$ on velocity and temperature is remarkable. Molar species concentration is accentuated with progressive values of Dufour number, while it is the opposite for velocity and temperature profiles. Moreover, thermal energy is absorbed due to the application of endothermic chemical reaction that cools the surroundings, which minimizes the diffusion rate of molecules and, therefore, less molar concentration is occurred. Conduction is the dominant mechanism for heat transport by applying Rayleigh number. The profiles of skin friction, Nusselt number, and Sherwood number are reduced with higher values of Rayleigh number. The present study has an appreciable impact on many engineering applications, such as magnetic storage media, the cooling systems of electronic devices, nuclear power plants, the chemical industry, and many more.
\end{abstract}

Keywords: Buoyancy ratio parameter, Chemical reaction, Heat source, Porous vertical cone, Viscous dissipation

$\begin{array}{cl}\text { Nomenclature } & \\ H_{0} & \text { magnetic field strength } \\ \mathbb{C} & \text { species concentration } \\ C_{\mathrm{r}} & \text { dimensional chemical reaction parameter } \\ C_{\mathrm{S}} & \text { concentration susceptibility } \\ \mathbb{C}_{\mathrm{W}} & \text { surface concentration } \\ \mathbb{C}_{\infty} & \text { free stream concentration } \\ D_{B} & \text { mass-diffusivity } \\ D_{T} & \text { coefficient of mass flux, through temperature gradient } \\ D u & \text { Dufour effect } \\ E c & \text { Eckert number } \\ \mathcal{F}^{\prime} & \text { non-dimensional velocity }\end{array}$

Walailak J Sci \& Tech 2021; 18(17): 22834 
$g \quad$ acceleration due to gravity

$\mathcal{G}$ non-dimensional temperature

$\mathcal{J}$ non-dimensional concentration

$k$ dimensional permeability parameter

$K \quad$ non-dimensional permeability parameter

$K_{r} \quad$ chemical reaction parameter

$K_{T} \quad$ thermal diffusion ratio

Le Lewis number

$M \quad$ magnetic parameter

$N_{b} \quad$ Brownian variable

$N_{r} \quad$ buoyancy ratio number

$N_{t} \quad$ thermophoretic parameter

Pr Prandtl number

$Q \quad$ non-dimensional heat source

$Q_{0} \quad$ dimensional heat source

$r \quad$ local radius of the cone

$\mathbb{T}$ dimensional nano-fluid temperature

$\mathbb{T}_{\mathrm{w}} \quad$ surface temperature

$\mathbb{T}_{\infty} \quad$ free stream temperature

$V_{0} \quad$ suction/injection parameter

\section{Greek Letters}

$\alpha_{f} \quad$ thermal diffusivity of base fluid

$\beta \quad$ coefficient of thermal expansion

$\psi \quad$ stream-function

$\zeta, \xi \quad$ cartesian coordinates

$\Gamma \quad$ non-dimensional similarity variable

$\gamma \quad$ semi-vertical angle of the cone

$\mu_{f} \quad$ viscosity at base fluid

$v_{f} \quad$ kinematic viscosity at base fluid

$\rho_{f} \quad$ density at base fluid

$\rho_{f_{\infty}} \quad$ free stream density at base fluid

$\rho_{p} \quad$ nanoparticle mass density

$\left(\rho C_{p}\right)_{f} \quad$ heat capacitance at base fluid

$\sigma \quad$ electric conductivity

$\Omega \quad$ dimensional velocity component along $\zeta$-axis

$\Omega_{w} \quad$ velocity at the surface of the cone 
http://wjst.wu.ac.th

\section{Introduction}

Nano-fluids are utilized as a heat transfer agent for their more developed thermal conductivity than base fluids and, thus, have assisted in a wide variety of imperative uses, such as vehicle engines, drug delivery, currency making, the cooling of electronics, etc. Usually, nanoparticles of metal, nonmetal, metal oxide, graphite, carbon nanotubes, and graphene are mixed with base fluids like water, ethylene glycol, lubricant oil, or blood to produce ensuing nano-fluids, and this notion of nanofluid is prompted by Choi [1]. Many experimental and theoretical inquiries have been carried out to identify the thermo-physical properties of nano-fluids, such as thermal-conductivity, and to consider their efficiency in reforming the performance of thermal and other devices.

In the last few years, due to the quick development of its applications, the study of heat and mass transport over a cone in a porous regime for a nano-fluid flow with a magnetic field has been the subject of attention of scientists, and a number of models have been testified to in the literature [2-4]. Natural convective flows of nano-fluids often arise in nature due to temperature and concentration differences, which have a perceptible impact on many applications of engineering, such as magnetic sensors and storage devices, the cooling organisms of electronic devices, and many more. Behseresht et al. [5] investigated the thermo-physical properties of nano-fluids for a vertical cone in porous medium and discussed the effect of thermophoretic force with Brownian motion in detail.

Due to the importance of extensive engineering applications, the behavior of nano-fluid flow in connection with suction or injection through heat and mass transport plays a vital role in the modern era. Nano-fluid of viscous incompressible flow over infinite permeable surface with suction or injection has gained extensive attention from many researchers, such as Mahdy [6]. Sameh et al. [7] conferred various formulae for viscosity and thermal conductivity for water-based nano-fluids having different nanoparticles over a truncated cone and achieved significant results. Chamkha et al. [8] explored the analysis of viscoelastic nano-fluid along a cone immersed in a porous medium due to uniform heat and volume-fraction fluxes. A rheological model for describing human blood flow over a permeable channel of CNTs was deliberated by Kalita et al. [9] for radiating fluid with the effects of magnetic drag force, as well as porosity, considering the effect of chemical reaction and heat generation. Raju et al. [10] presented the behavior of a rotating cone for porosity with the effect of heat source and sink.

Rayleigh number is a dimensionless number, with the help of which we can easily check whether a natural convection boundary layer is laminar or turbulent. The importance of Hartmann number and Rayleigh number in free convective nano-fluid flow with MHD and viscosity dependent on magnetic field was reported by Sheikholeslami et al. [11]. Thermal-radiation on momentum and heat transfer processes is also a theme of attentiveness for its applications, for instance, photo chemical reactors, nuclear power plants, and various devices for aircraft, missiles, satellites, and space vehicles. The impact of radiation and magnetic field on nano-fluid flow over a vertical cone has been investigated by many researchers [12-14]. Also, Rashad et al. [15] presented the impact of radiation on nano-fluid flow over a permeable cone and detected the prominence of Brownian motion parameter in Nusselt number and Sherwood number.

Reddy et al. [16] reported the effect of natural convection on a chemically reacting fluid of nanoparticles over a vertical cone. The investigation of a chemically reacting nano-fluid flow of convective heat and mass transport has suitable uses in many branches of engineering, like industries related to chemical and petroleum, nuclear reactor cooling, and packed-bed catalytic reactors [17-19].

Various models have been implemented with the mutual effect of motion of nanoparticles and thermos diffusion and solved numerically by using different suitable numerical techniques. Thermophoresis effect is the transport force generated by the presence of a temperature gradient. The relation between nanoparticle diffusion due to the thermophoresis effect and the thermal diffusion within a nano-fluid is known as Thermophoresis parameter. Many researches [20-22] have been accomplished with these powerful effects and useful conclusions have been made. Moreover, Soret number is significant where the existence of more than one chemical species is considered under higher temperature gradients. The effect of viscous dissipation is important in several applied uses, such as the transportation of oil products through channels, the processing of polymers, etc. Usually, this outcome is categorized by 
http://wjst.wu.ac.th

the Eckert number, and the effect has been examined by many authors. With the applications of viscous dissipation, magnetic drag force, and thermo diffusion on convective heat-mass transport, Hazarika et al. [23] inspected the impacts for different nanoparticles.

Recently, Sheikholeslami et al. [28-31] discussed the thinner boundary layer by the insertion of helical tape. They used copper oxide nanoparticles to enhance the thermal features. Moreover, they presented 2 kinds of flat plate collectors, and utilized nano-fluids in such collectors, which gave higher thermal conductivity. Further, they used the turbulators to enhance the nano-fluid flow, resulting in higher heat transfer, which also gave maximum flow disturbance. After that, they investigated a combination of helical coil and twisted tape within a 6-lobed tube using a hybrid nano-fluid. The effect of thermal conductivity and variable viscosity along an isothermal vertical surface was discussed by Hazarika and Ahmed [32], who found that the progress of thermal-radiation increases gas temperature.

The objective of this investigation is to assess the natural convective MHD flow of nano-fluid over a vertical cone surrounded by porous regime. The governing PDEs are reduced to a system of coupled ODEs by utilizing similarity transform, and then MATLAB bvp4c code is developed to solve them numerically. Based on the above literature, and to the best of author's knowledge, no studies have been documented describing the effect of endothermic and exothermic reaction with heat source/sink on convective MHD nano-fluid flow over a vertical cone. Therefore, we have tried to emphasize these 2 parameters in our present study and have found significant outcomes. Moreover, the behavior of the other key parameters, like thermophoretic force, Dufour number, Brownian motion parameter, and buoyancy ratio parameter on momentum, energy, and mass equations are discussed and elaborated on and plotted graphically.

\section{Materials and methods}

In this investigation, we have presented a flow model in Figure 1 for steady 2-dimensional nanofluid over a vertical cone immersed in a porous media. A uniform identical magnetic field of strength $H_{0}$ is applied normal to the surface of the cone. The $\zeta$ is chosen in the way of flow over the cone surface, and $\xi$ is booked normal to it; local radius is taken as $r$, and $\gamma$ is the semi-vertical angle of the cone. According to the influence of the Brownian motion and thermophoresis, the nanoparticles are induced in the base fluid.

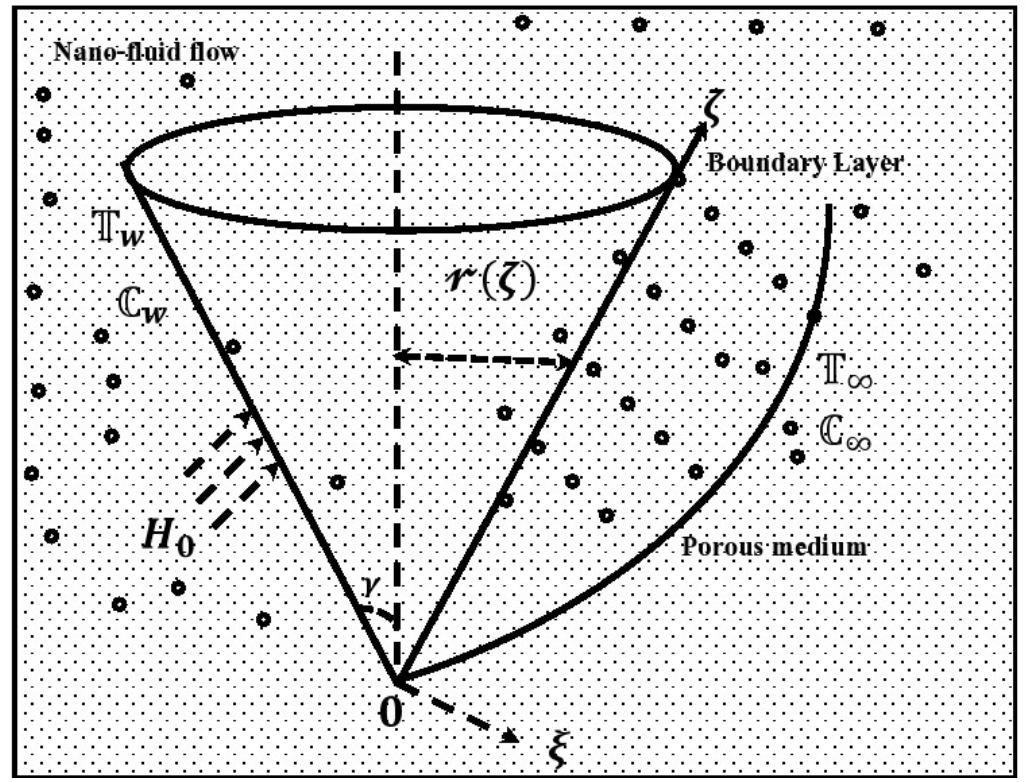

Figure 1 Geometry of flow model. 
The primary equations of momentum, energy, and mass transfer for nano-fluids in the presence of other significant parameters are as follows;

$(r \Omega)_{\zeta}+(r \Delta)_{\xi}=0$

$\Omega(\Omega)_{\zeta}+\Delta(\Delta)_{\xi}=\left[\begin{array}{c}v_{f}(\Omega)_{\xi \xi}-\frac{\sigma H_{0}^{2}}{\rho_{f}} \Omega-\frac{\mu_{f}}{k \rho_{f}} \Omega \\ +\mathrm{g} \cos \gamma\left\{\left(1-\mathbb{C}_{\infty}\right) \rho_{f_{\infty}} \beta\left(\mathbb{T}-\mathbb{T}_{\infty}\right)-\left(\rho_{p}-\rho_{f_{\infty}}\right)\left(\mathbb{C}-\mathbb{C}_{\infty}\right)\right\}\end{array}\right]$

$\Omega(\mathbb{T})_{\zeta}+\Delta(\mathbb{T})_{\xi}=\left[\begin{array}{c}\alpha_{f}(\mathbb{T})_{\xi \xi}+\frac{\mu_{f}}{\left(\rho c_{p}\right)_{f}}\left(\Omega_{\xi}\right)^{2}+\left(\frac{D_{B} K_{T}}{\left(\rho c_{p}\right)_{f} C_{S}}\right)(\mathbb{C})_{\xi \xi} \\ +Q_{0}\left(\mathbb{T}-\mathbb{T}_{\infty}\right)+\tau\left\{D_{B}(\mathbb{C})_{\xi}(\mathbb{T})_{\xi}+\left(\frac{D_{T}}{T_{\infty}}\right)\left(\mathbb{T}_{\xi}\right)^{2}\right\}\end{array}\right]$

$\Omega(\mathbb{C})_{\zeta}+\Delta(\mathbb{C})_{\xi}=D_{B}(\mathbb{C})_{\xi \xi}+\left(\frac{D_{T}}{T_{\infty}}\right)(\mathbb{T})_{\xi \xi}-C_{r}\left(\mathbb{C}-\mathbb{C}_{\infty}\right)$

The boundary conditions are;

$$
\left\{\left(\begin{array}{c}
\left.\Omega=\Omega_{w}=\frac{\alpha_{f}\left(\Re_{a \zeta}\right)^{\frac{1}{2}}}{\zeta}, \Delta=\Delta_{w},\right) \text { at } \xi=0 \\
\mathbb{T}=\mathbb{T}_{w}, \mathbb{C}=\mathbb{C}_{w} \\
\Omega=0, \mathbb{T} \rightarrow \mathbb{T}_{\infty}, \mathbb{C} \rightarrow \mathbb{C}_{\infty} \text { as } \xi \rightarrow \infty
\end{array}\right\}\right.
$$

The similarity transforms are defined as;

$$
\left\{\begin{array}{l}
\Gamma=\frac{\xi}{\zeta}\left(\Re_{a_{\zeta}}\right)^{\frac{1}{4}}, \quad \mathcal{F}(\Gamma)=\frac{\psi}{r\left(\Re_{a_{\zeta}}\right)^{\frac{1}{4}}}, \\
\mathcal{G}(\Gamma)=\frac{\mathbb{T}-\mathbb{T}_{\infty}}{\mathbb{T}_{w}-\mathbb{T}_{\infty}}, \quad \mathcal{J}(\Gamma)=\frac{\mathbb{C}-\mathbb{C}_{\infty}}{\mathbb{C}_{w}-\mathbb{C}_{\infty}}
\end{array}\right\}
$$

We introduce stream function $\psi(x, y)$, which satisfies the equation of continuity (1), defined as; $\Omega=r^{-1}(\mathbb{Z})_{\xi}$ and $\Delta=-r^{-1}(\mathbb{Z})_{\zeta}$

The dimensionless parameters are; 


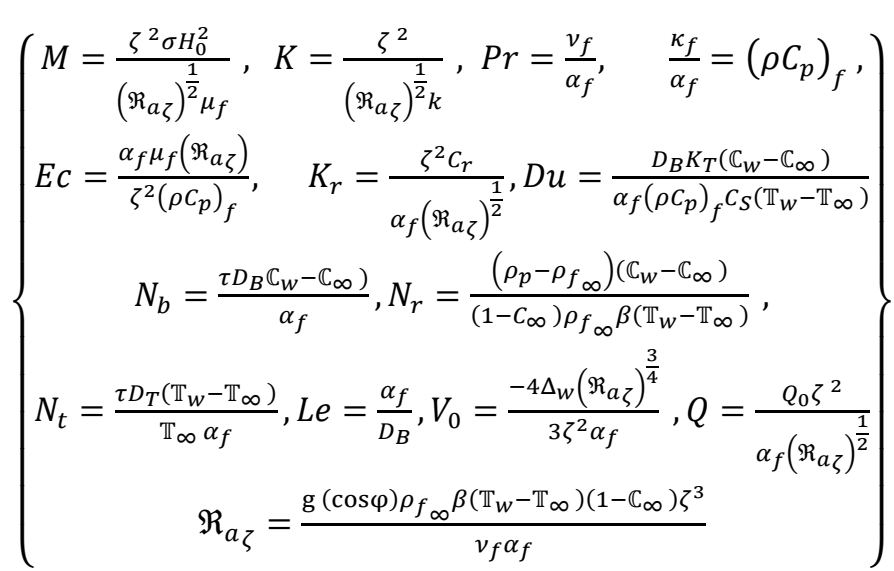

In view of the similarity transformations (6) to (8), the Eqs. (2) - (4) become;

$\mathcal{F}^{\prime \prime \prime}-\frac{1}{P r}\left\{\frac{1}{2} \mathcal{F}^{\prime 2}-\frac{3}{4} \mathcal{F} \mathcal{F}^{\prime \prime}\right\}+\left(\mathcal{G}-N_{r} \mathcal{J}\right)-(K+M) \mathcal{F}^{\prime}=0$,

$\mathcal{G}^{\prime \prime}-\frac{\Gamma}{4} \mathcal{F}^{\prime} \mathcal{G}^{\prime}+\frac{3}{4} \mathcal{F} \mathcal{G}^{\prime}+N_{b} \mathcal{G}^{\prime} \mathcal{J}^{\prime}+N_{t} \mathcal{G}^{\prime 2}+D u \mathcal{J}^{\prime \prime}+E c \mathcal{F}^{\prime \prime 2}-Q \mathcal{G}=0$

$\mathcal{J}^{\prime \prime}-\frac{\Gamma}{4} \mathcal{F}^{\prime} \mathcal{J}^{\prime}+\frac{3}{4} \mathcal{F} \mathcal{J}^{\prime}+\frac{N_{t}}{N_{b}} \mathcal{G}^{\prime \prime}+L e K_{r} \mathcal{J}=0$

Eq. (5) reduces to;

$$
\left\{\begin{array}{c}
\mathcal{F}(0)=V_{0}, \mathcal{F}^{\prime}(0)=1, \mathcal{G}(0)=1, \mathcal{J}(0)=1, \\
\mathcal{F}^{\prime}(\Gamma) \rightarrow 0, \quad \mathcal{G}(\Gamma) \rightarrow 0, \mathcal{J}(\Gamma) \rightarrow 0 \text { as } \Gamma \rightarrow \infty
\end{array}\right\}
$$

The coefficient of velocity, temperature, and species concentration gradients at the surface in dimensional form are defined as;

$\bar{C}_{f}=\frac{2 \tau_{w}}{\rho_{f} \Omega_{w}^{2}}, \quad \overline{N u}_{\xi}=\frac{\zeta q_{m}}{\kappa_{f}\left(\mathbb{T}_{w}-\mathbb{T}_{\infty}\right)}, \quad \overline{S h}_{\xi}=\frac{\zeta J_{w}}{D_{B}\left(\mathbb{C}_{w}-\mathbb{C}_{\infty}\right)}$

where $\tau_{w}, q_{m}$, and $J_{w}$ represent the shear stress, heat flux, and mass flux at the surface, respectively, and are defined as;

$$
\begin{gathered}
\tau_{w}=\left.\mu_{n f}(\Omega)_{\xi}\right|_{\xi=0} \\
q_{m}=-\left.\kappa_{n f}(\mathbb{T})_{\xi}\right|_{\xi=0} \\
J_{w}=-\left.D_{B}(\mathbb{C})_{\xi}\right|_{\xi=0}
\end{gathered}
$$

Using the Eqs. (14) - (16), the coefficient of velocity, temperature, and species concentration gradients at the surface in non-dimensional form are; 


$$
\begin{aligned}
& C_{f}=\bar{C}_{f}\left(\Re_{a \zeta}\right)^{\frac{1}{2}}=2 \operatorname{Pr}\left(\Re_{a_{\zeta}}\right)^{\frac{1}{4}} \mathcal{F}^{\prime \prime}(0), \\
& N u_{\xi}=\overline{N u}_{\xi}\left(\Re_{a_{\zeta}}\right)^{-\frac{1}{2}}=-\left(\Re_{a_{\zeta}}\right)^{-\frac{1}{4}} \mathcal{G}^{\prime}(0), \\
& S h_{\xi}=\overline{S h}_{x}\left(\Re_{a_{\zeta}}\right)^{-\frac{1}{2}}=-\left(\Re_{a_{\zeta}}\right)^{-\frac{1}{4}} \mathcal{J}^{\prime}(0),
\end{aligned}
$$

where $\Re_{a \zeta}$ is the Rayleigh number.

Table 1 Velocity distribution for $M$.

\begin{tabular}{ccccc}
\hline & \multicolumn{2}{c}{$\begin{array}{c}\text { Chamkha et al. } \mathbf{~ [ 1 2 ]} \\
\text { at } \boldsymbol{Q}=\mathbf{0}\end{array}$} & \multicolumn{2}{c}{$\begin{array}{c}\text { Present model } \\
\text { at } \boldsymbol{Q}=\mathbf{0 . 2}\end{array}$} \\
\cline { 2 - 5 } & \multicolumn{2}{c}{$\mathbf{D u}$} & $\mathbf{2}$ & $\mathbf{D u}$ \\
\hline $\boldsymbol{\Gamma}$ & $\mathbf{0 . 1}$ & $\mathbf{0 . 3}$ & 1.00000 & $\mathbf{0 . 3}$ \\
$\mathbf{0 . 0}$ & 1.00000 & 1.00000 & 0.34228 & 1.00000 \\
$\mathbf{1 . 0}$ & 0.36306 & 0.39057 & 0.11425 & 0.38101 \\
$\mathbf{2 . 0}$ & 0.13163 & 0.16601 & 0.03597 & 0.13761 \\
$\mathbf{3 . 0}$ & 0.044891 & 0.063115 & 0.010329 & 0.054588 \\
$\mathbf{4 . 0}$ & 0.016593 & 0.021126 & 0.000000 & 0.018119 \\
$\mathbf{5 . 0}$ & 0.000000 & 0.000000 & & 0.000000 \\
\hline
\end{tabular}

\section{Validity and accuracy}

To check the validity of the currently employed numerical scheme, the numerical outputs are compared with those developed by Chamkha et al. [12] on variation of velocity for $D u$ over the surface of the cone, and we attain a very noble agreement. Table 1 justifies that our results as being genuine and well validated.

Table 2 Stability test for the proposed investigation of velocity, temperature, and concentration.

\begin{tabular}{cccccccccc}
\hline & \multicolumn{3}{c}{$\mathcal{F}^{\prime}(\boldsymbol{\Gamma})$} & \multicolumn{3}{c}{$\mathcal{G}(\boldsymbol{\Gamma})$} & \multicolumn{3}{c}{$\mathcal{J}(\boldsymbol{\Gamma})$} \\
\cline { 2 - 11 } & \multicolumn{9}{c}{$\mathbf{d}$ (step size) } \\
\hline $\boldsymbol{\Gamma}$ & $\mathbf{0 . 0 1}$ & $\mathbf{0 . 0 0 1}$ & $\mathbf{0 . 0 0 0 1}$ & $\mathbf{0 . 0 1}$ & $\mathbf{0 . 0 0 1}$ & $\mathbf{0 . 0 0 0 1}$ & $\mathbf{0 . 0 1}$ & $\mathbf{0 . 0 0 1}$ & $\mathbf{0 . 0 0 0 1}$ \\
\hline 0.0 & 1.000000 & 1.000000 & 1.000000 & 1.000000 & 1.000000 & 1.000000 & 1.000000 & 1.000000 & 1.000000 \\
1.0 & 0.473123 & 0.399063 & 0.237358 & 0.448021 & 0.331272 & 0.297135 & 0.481072 & 0.391085 & 0.315101 \\
2.0 & 0.216033 & 0.199139 & 0.100514 & 0.271086 & 0.221850 & 0.180105 & 0.319417 & 0.270221 & 0.209381 \\
3.0 & 0.106682 & 0.099974 & 0.047595 & 0.190564 & 0.102445 & 0.061844 & 0.204187 & 0.174520 & 0.127324 \\
4.0 & 0.057096 & 0.008781 & 0.003219 & 0.099105 & 0.058411 & 0.010254 & 0.091462 & 0.045050 & 0.018084 \\
5.0 & 0.001076 & 0.000305 & 0.000207 & 0.007912 & 0.006481 & 0.000381 & 0.005291 & 0.002853 & 0.001057 \\
6.0 & 0.000030 & 0.000051 & 0.000030 & 0.000530 & 0.000105 & 0.000104 & 0.000502 & 0.000201 & 0.000015 \\
7.0 & 0.000000 & 0.000000 & 0.000000 & 0.00000 & 0.000000 & 0.000000 & 0.000000 & 0.000000 & 0.000000 \\
\hline
\end{tabular}


http://wjst.wu.ac.th

\section{Stability and convergence}

In Table 2, we observe the stability of the numerical scheme over the grid invariance test for the nano-fluid velocity, temperature, and nanoparticle concentration. When we reduce the step size, as well as increase the number of elements in the same domain, the stability remains unchanged. This shows that the implemented scheme is feasible and, so, it is stable and convergent.

\section{Results and discussion}

In the present research, MHD natural convective flow of chemically reacting nano-fluid with Brownian motion, thermophoresis, heat source/sink, porosity, and viscous dissipation over a vertical cone is discussed. For all the graphs of $\mathcal{F}^{\prime}(\Gamma), \mathcal{G}(\Gamma)$, and $\mathcal{J}(\Gamma)$ of nano-fluid, the default parameters are $M=2, K=0.3, P r=0.72, Q=0.15, N_{r}=0.5, N_{t}=0.5, K_{r}=0.02, E c=0.04, D u=0.2, L e=0.5$, and otherwise indicated.

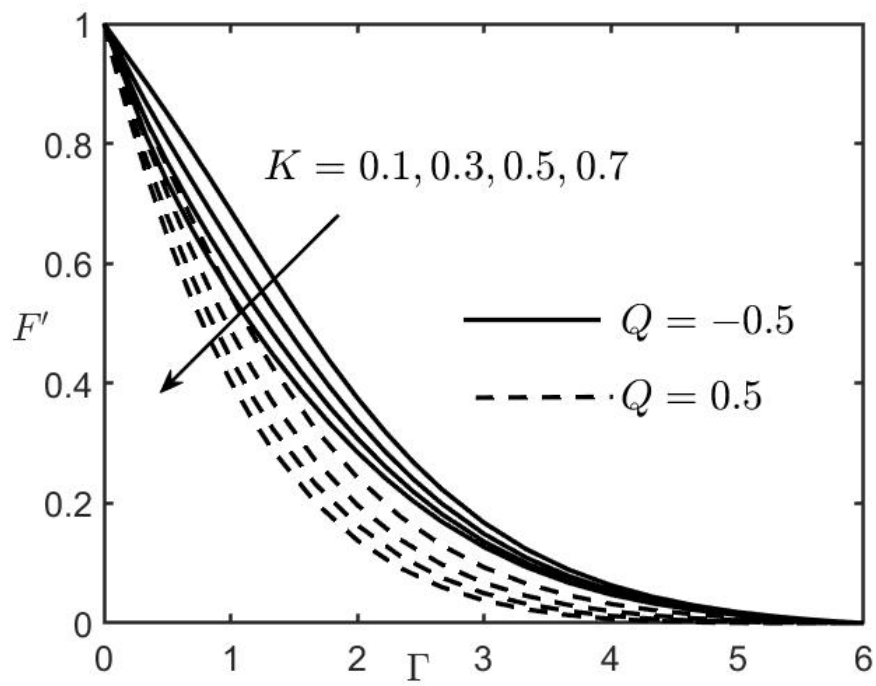

Figure 2 Variation of $K$ and $Q$ over $\mathcal{F}^{\prime}$.

Figure 2 highlights the behavior of porosity $(K)$ of the porous medium and heat generation/absorption $(Q)$ on nano-fluid velocity $\left(\mathcal{F}^{\prime}\right)$. Porosity of a porous medium is a measurement of its ability to hold a fluid, whereas permeability is the ability of fluid to ease movement of molecules through the medium. The porosity of the medium may be calculated as a fraction of the volume of voids over the total volume, between 0 and 1 , or as a percentage, between 0 and $100 \%$. The porosity of a medium acts as a resistive force to the motion of molecules of the fluid and, therefore, the curves of velocity decline throughout the motion. An asymptotic behavior is perceived, and $\mathcal{F}^{\prime}$ is least, at higher porosity $K=0.7$, as the drag-force of porosity delays the motion of the particles, so the thickness of the momentum boundary layer is diminished. The transfer of heat in fluid mechanics by convection signifies that the motion of the fluid molecules carries heat from one molecule to another molecule. However, heat may be transferred by conduction, which does not involve any motion of a mass of fluid, but rather is a transfer of energy within a mass of fluid or between masses in contact. The term $Q_{0}\left(\mathbb{T}-\mathbb{T}_{\infty}\right)$ in the energy Eq. (3) represents the amount of heat generation or absorption per unit volume, where $Q_{0}>0$ represents source and $Q_{0}<0$ represents sink. $Q_{0}>0$ implies that $\mathbb{T}>\mathbb{T}_{\infty}$, which leads to heat being conveyed from the surface of the cone to the free stream and, therefore, the energy in the form of heat resists the motion of fluid molecules which carries the energy from the surface of the cone to the 
http://wjst.wu.ac.th

free stream. $Q<0$ implies that $\mathbb{T}_{\infty}>\mathbb{T}$, which leads to diffusion of the heat from the free stream to the surface of the cone and, so, the molecules absorb energy in the form of heat, that leads to free movement of molecules towards the free stream. Therefore, the curves of $\mathcal{F}^{\prime}$ have higher values due to heat sink $(Q=-0.5)$ and have a lower value for the heat source $(Q=0.5)$.

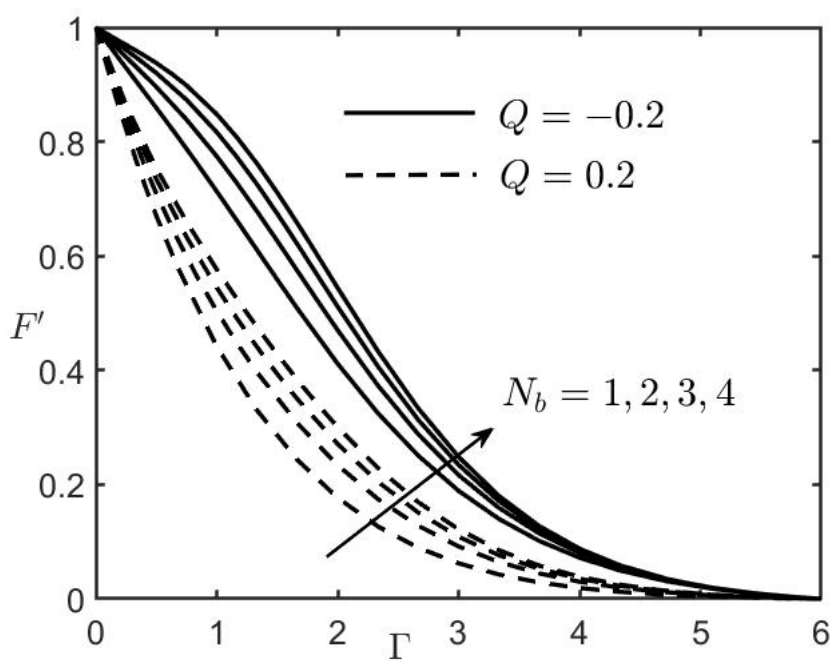

Figure 3 Variation of $N_{b}$ and $Q$ over $\mathcal{F}^{\prime}$.

Figure 3 portrays the influence of Brownian motion $\left(N_{b}\right)$ and heat source/sink $(Q)$ on nano-fluid velocity. In fluid mechanics, particles in both liquids and gases move randomly due to bombardment by the other moving particles in the fluid, and this phenomenon is called Brownian motion, where larger particles can be moved by light and fast-moving molecules of the fluid. It is the random motion of particles suspended in a fluid medium and was named after the Scottish botanist Robert Brown [26], the first to study such fluctuations of particle position inside a fluid sub-domain. Due to Brownian motion, the fluid molecules move faster from the surface to the free stream and, therefore, the curves of velocity are elevated in the presence of heat source and sink. As earlier discussed, the behavior of $Q$ in Figure 2 is such that $Q<0$ gives the higher values in $\mathcal{F}^{\prime}$, and lower values are observed in $\mathcal{F}^{\prime}$ for $Q>0$. 
http://wjst.wu.ac.th

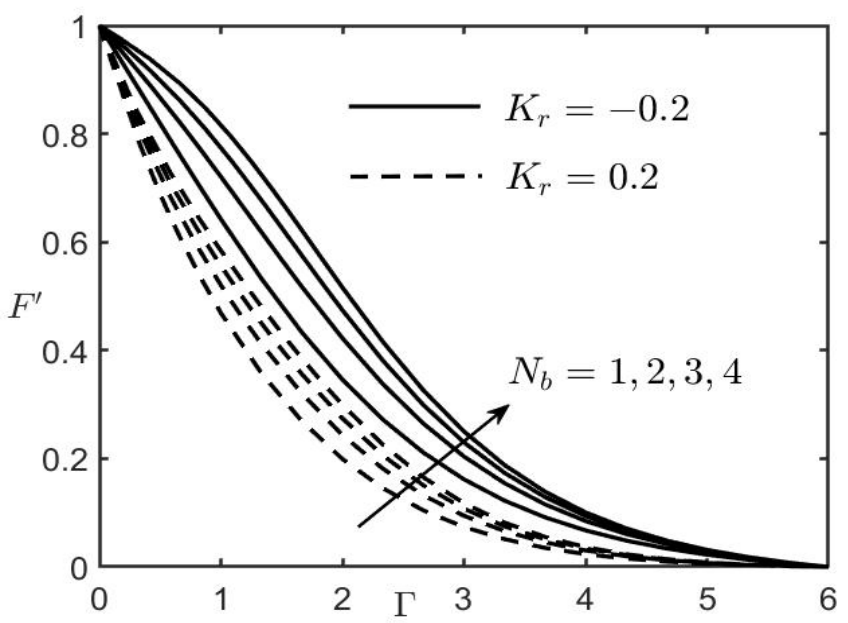

Figure 4 Variation of $N_{b}$ and $K_{r}$ over $\mathcal{F}^{\prime}$.

The behavior of endothermic $\left(K_{r}>0\right)$ and exothermic $\left(K_{r}<0\right)$ chemical reactions over the nanofluid velocity in the presence of Brownian motion effect $\left(N_{b}\right)$ is displayed in Figure 4. When a chemical reaction happens, thermal energy is transferred to or from the surroundings. When the thermal energy is transferred to the surroundings, this is called an exothermic reaction, and the temperature of the surroundings increases. Endothermic reactions take place in thermal energy and the temperature of the surroundings decreases. In endothermic chemical reactions, a positive change in enthalpy is reckoned, while a negative change in enthalpy may occur in exothermic chemical reactions. The growth of $N_{b}$ leads to a rise in the thickness of the boundary layer; thus, the profiles of $\mathcal{F}^{\prime}$ are enhanced for $N_{b}=4$ but, due to the effect of $K_{r}=0.2$, a fall in $\mathcal{F}^{\prime}$ is noticed. In this study, it is seen that the transition of the rate of mass flux means that movement of the molecules are from endothermic to exothermic processes and, hence, velocity declines due to the effect of chemical reaction.

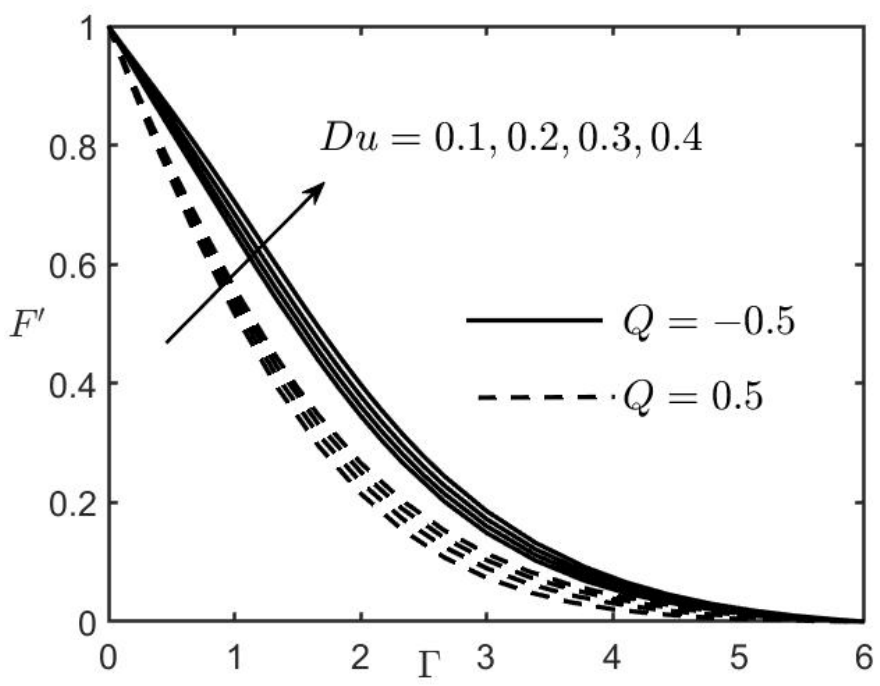

Figure 5 Variation of $D u$ and $Q$ over $\mathcal{F}^{\prime}$. 
http://wjst.wu.ac.th

In Figure 5, the outlines are drawn to explore the effect of Dufour $(D u)$ along with heat source $(Q>0)$ and heat $\operatorname{sink}(Q<0)$ over the nano-fluid velocity $\left(\mathcal{F}^{\prime}\right)$. Dufour [24] was the first observer who had deliberated the Dufour effect $(D u)$, which is a cross diffusion effect due to simultaneous occurrence of heat and mass transfer in a moving fluid affecting each other. The non-dimensional Dufour number $(D u)$ is represented as $D u=$ (contribution of the concentration gradients) / (thermal energy flux) and it is termed the diffusion thermo. The trends of concentration gradient are to create the energy flux, which changes the temperature of the nano-fluid. The application of Dufour enhances the velocity $\left(\mathcal{F}^{\prime}\right)$ due to the higher thermal energy flux. Sink uplifts the values of $\mathcal{F}^{\prime}$, while heat source diminishes the fluid velocity.

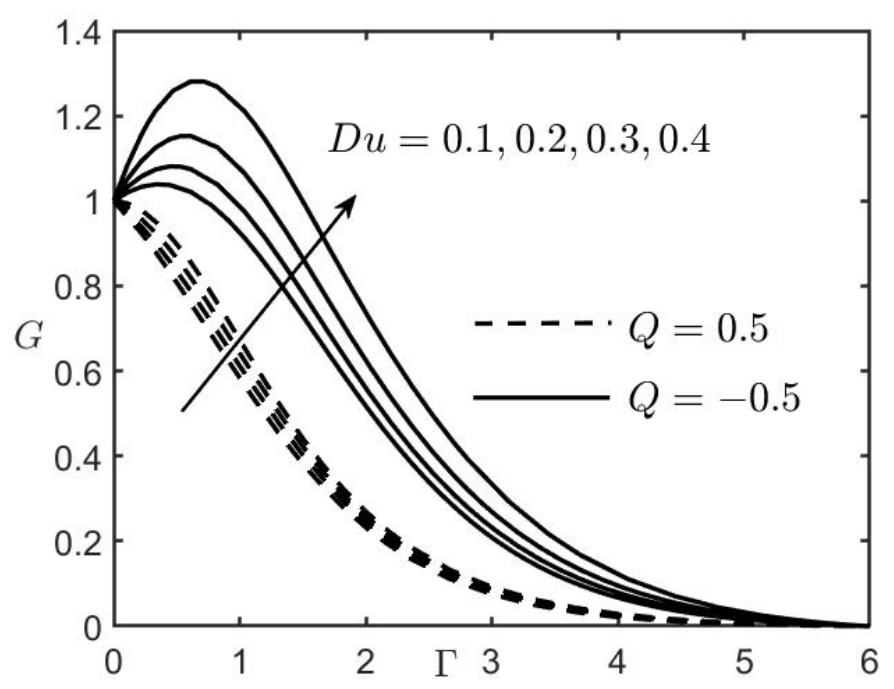

Figure 6 Variation of $D u$ and $Q$ over $\mathcal{G}$.

Figure 6 depicts the impact of heat source $(Q>0)$ and heat $\operatorname{sink}(Q<0)$ on the application of Dufour effect $(D u)$ on the outlines of the fluid temperature $(\mathcal{G})$. In this study, $D u=0.1,0.2,0.3,0.4$ (< 1) indicates that thermal energy flux dominant over the concentration gradients and the higher thermal energy boosts the temperature of the nano-fluid for the augmented values of $D u$. The heat source $(Q=$ $0.5)$ reduces the fluid temperature. A peak elevation is noticed for heat $\operatorname{sink}(Q=-0.5)$ near $0 \leq \Gamma \leq 2$ due to generated heat from the surface of the cone in the fluid medium and, hence, the thickness of the thermal boundary layer upsurges. It is concluded that heat is transferred from a higher temperature to a lower temperature and, hence, temperature and thermal boundary layer thickness decline due to the impact of heat source and sink. 
http://wjst.wu.ac.th

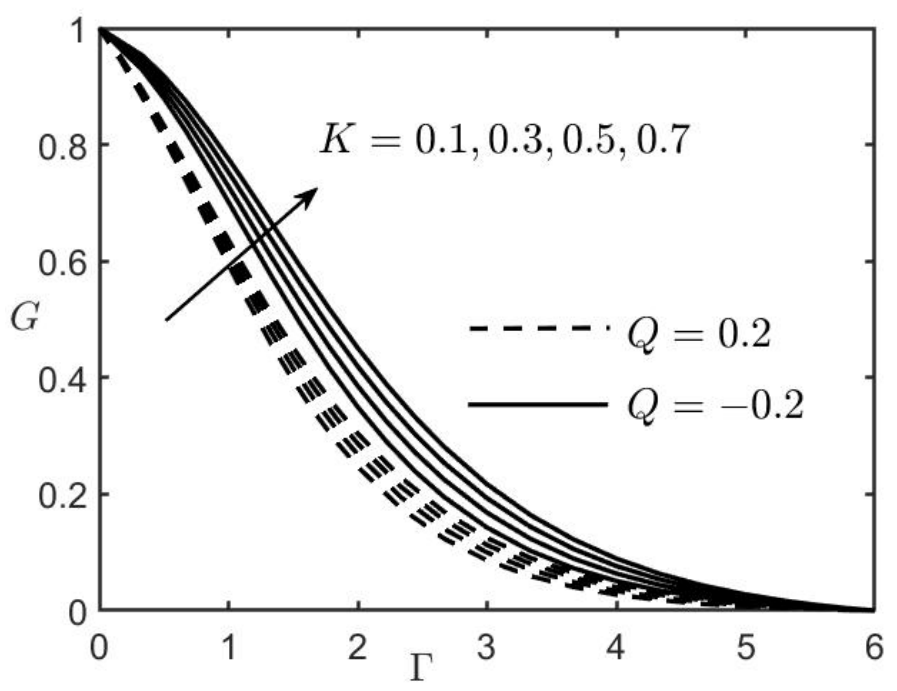

Figure 7 Variation of $K$ and $Q$ over $\mathcal{G}$.

Figure 7 shows the response of porosity of the medium $(K)$ in the presence of heat source $(Q>0)$ and heat $\operatorname{sink}(Q<0)$ on the contours of fluid temperature $(\mathcal{G})$. Physically, $K$ is the measurement of void spaces in a material; it can be termed as $K=$ ( volume of pores) / (volume of bulk solid bodies) and is usually expressed as a percentage; porosity $=($ Volume of Voids / Total Volume $) \times 100 \%$. In this investigation, the porosity is based on Darcy's law [25]. The porosity always behaves like a drag force which resists the motion of convective molecules in the thermal boundary layers, and that implicates the increase in heat which leads to boosting the fluid temperature $(\mathcal{G})$. Higher temperature occurs due to heat $\operatorname{sink}(Q=-0.2)$, and temperature falls towards the surface of the cone by heat source $(Q=0.2)$.

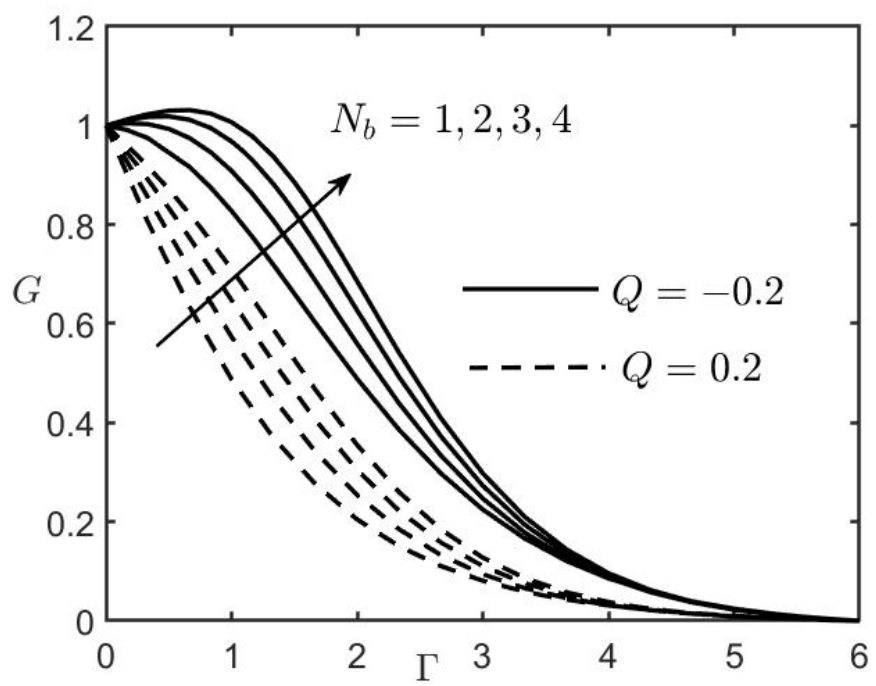

Figure 8 Variation of $N_{b}$ and $Q$ over $\mathcal{G}$. 
http://wjst.wu.ac.th

Contours of fluid temperature $(\mathcal{G})$ for the Brownian motion $\left(N_{b}\right)$ and heat source/sink $(Q)$ are portrayed in Figure 8. The thermal motion is a phenomenon that consists of the continuous random movement of atoms and molecules in a liquid or gas due to thermal energy, which produces the Brownian motion [26]. In lower viscous molecules, with smaller size of molecules, and with higher temperature, the Brownian motion may develop. The Brownian motion parameter $\left(N_{b}\right)$ is the ratio of the nanoparticle diffusion due to the Brownian motion effect to the thermal diffusion in the nano-fluid. According to the Einstein-Stokes equation (Buongiorno, [27]), the Brownian motion is proportional to the inverse of the particle diameter and, hence, the Brownian motion can be enhanced when the diameter of the particle is augmented. Therefore, the augmented $N_{b}$ enhances the fluid temperature and its width of thermal layers in the presence of heat source and sink. Nano-fluid temperature is minimum for $Q=0.2$ than that of $Q=-0.2$, and a beautiful asymptotic view is observed after $\Gamma \leq 4$.

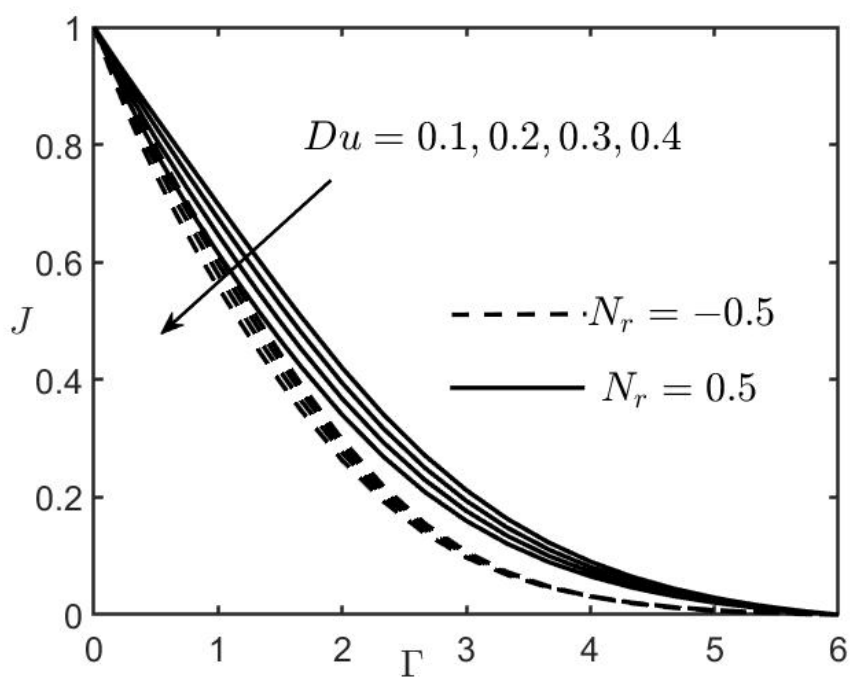

Figure 9 Variation of $D u$ and $N_{r}$ over $\mathcal{J}$.

In Figure 9, the outlines of species concentration $(\mathcal{J})$ for Dufour effect $(D u)$ and buoyancy ratio $\left(N_{r}\right)$ have been exhibited over a vertical cone. Outlines of $\mathcal{J}$ are reduced for higher values of $D u=0.4$ and, thus, the thickness of concentration boundary layer becomes minimum. Here, $N_{r}=$ (Buoyancy force due to mass transfer) / (Buoyancy force due to heat transfer). When $N_{r}>0$, the buoyancy force due to mass transfer dominates over the buoyancy force, due to heat transfer that leads to uplifting of the species concentration, meaning faster diffusion of molecules may occur. Moreover, for $N_{r}<0$, the buoyancy force due to heat transfer dominates over the buoyancy force due to mass transfer, which slows diffusion of molecules that may occur in the molar concentration layers and, thus, the species concentration declines. 
http://wjst.wu.ac.th

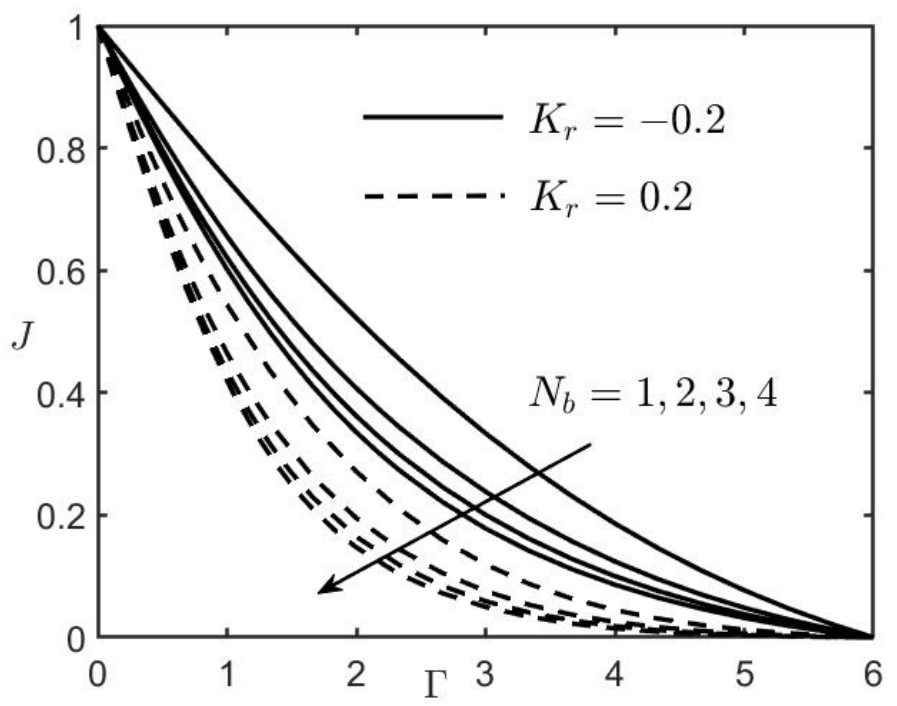

Figure 10 Variation of $N_{b}$ and $K_{r}$ over $\mathcal{J}$.

Figure 10 illustrates the profiles of species concentration $(\mathcal{J})$ for Brownian motion $\left(N_{b}\right)$ in the presence of endothermic $\left(K_{r}>0\right)$ and exothermic $\left(K_{r}<0\right)$ chemical reactions. As we know, Brownian motion instigates the nanoparticles to transfer from the upper surfaces of species concentration to the lesser surfaces of species concentration, which leads to decline in the profiles of $\mathcal{J}$ for higher values of $N_{b}$. Consequently, a species concentration boundary layer of nanoparticles occurs at the surface of the cone. During exothermic chemical reaction $\left(K_{r}=-0.2\right)$, the thermal energy is released from the surface of the cone to the fluid medium and, therefore, the molar concentration gains. The thermal energy is absorbed due to the application of endothermic chemical reaction $\left(K_{r}=0.2\right)$ that cools the surroundings, which involves loss of the diffusion rate of molecules and, thereby, lower molar concentration occurs.

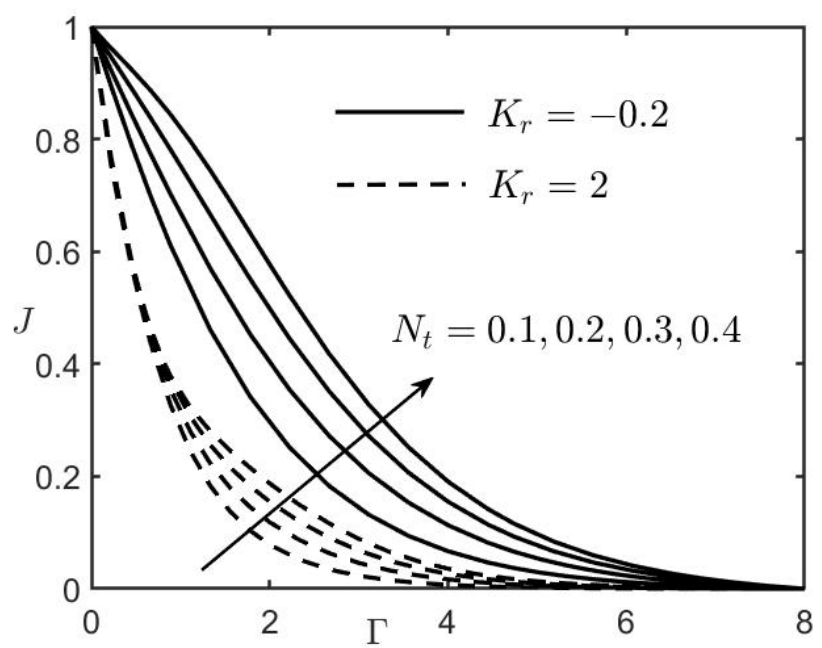

Figure 11 Variation of $N_{t}$ and $K_{r}$ over $\mathcal{J}$. 
http://wjst.wu.ac.th

The effect of thermophoresis $\left(N_{t}\right)$ and endothermic/exothermic chemical reactions $\left(K_{r}\right)$ on the contours of species concentration $(\mathcal{J})$ is discussed in Figure 11. The phenomenon of thermophoresis may exist in the combinations of mobile particles, in which the force of a temperature gradient can be observed due to the different responses given by different types of particles. In the physical point of view, thermophoretic force $\left(N_{t}\right)$ generates the temperature gradient between the heated fluid and the non-heated surface, which affects the movement of nanoparticles near the surface. Therefore, $N_{t}$ generates the elevated curves and develops the growth of molar species layers. Growth values of $N_{t}$ lead to rise in the profiles of $\mathcal{J}$ and the solutal boundary layer of nanoparticles rises over the cone surface. In addition, $K_{r}=-0.2$ is more dominant over $K_{r}=2.0$. At $\Gamma \leq 1$, the boundary layer is seen to be very thick; after that, it increases exponentially due to the presence of $K_{r}=2.0$.

The velocity-gradient, temperature-gradient, and species concentration-gradient at the surface of the cone for magnetic drag force $(M)$ and heat generation parameter $(Q)$ against Rayleigh number $\left(\Re_{a_{\zeta}}\right)$ are presented in Tables 3 - 5, respectively. The Rayleigh number $\left(\Re_{a_{\zeta}}\right)$ is associated with buoyancy-driven airflow and can be regarded as a measure of the driving forces of natural convection. It is used to express heat transfer in natural convection. The magnitude of the Rayleigh number is a good indication as to whether the natural convection boundary layer is laminar or turbulent. Therefore, as $\mathfrak{R}_{a_{\zeta}}$ grows, $C_{f}, N u_{\xi}$, and $S h_{\xi}$ decline due to the convective heat transfer which leads to free movement of the molecules. The reduction in Nusselt number $\left(N u_{\xi}\right)$ is more pronounced for higher values of heat source $(Q)$ and Hartmann number $(M)$, while Sherwood number $\left(S h_{\xi}\right)$ behaves opposite. It is an interesting observation that increasing Lorentz forces declines the effect of skin friction coefficient $\left(C_{f}\right)$, as well as Nusselt number $\left(N u_{\xi}\right)$. Increasing Rayleigh number $\left(\mathfrak{R}_{a_{\zeta}}<1\right)$ from $\frac{1}{10}$ up to $\frac{4}{10}$ leads to increase in the viscous forces and overcomes the buoyancy forces and, therefore, convection currents dominate at low Rayleigh numbers.

Table 3 Skin friction coefficient.

\begin{tabular}{ccccccc}
\hline \multicolumn{7}{c}{$\mathbf{2} \boldsymbol{P r}\left(\boldsymbol{R}_{\boldsymbol{a}_{\zeta}}\right)^{\frac{\mathbf{1}}{\mathbf{4}}} \mathcal{F}^{\prime \prime}(\mathbf{0})=\boldsymbol{C}_{\boldsymbol{f}}$} \\
\hline $\boldsymbol{R}_{\boldsymbol{a \zeta}_{\boldsymbol{C}}}$ & $\boldsymbol{M}=\mathbf{0 . 0}$ & $\boldsymbol{M}=\mathbf{2 . 0}$ & $\boldsymbol{M}=\mathbf{4 . 0}$ & $\boldsymbol{Q}=\mathbf{0 . 0}$ & $\boldsymbol{Q}=\mathbf{0 . 2}$ & $\boldsymbol{Q}=\mathbf{0 . 5}$ \\
\hline 0.1 & -0.7705 & -1.412 & -1.8843 & -1.6029 & -1.4825 & -1.4125 \\
0.2 & -0.9163 & -1.679 & -2.2408 & -1.9062 & -1.7631 & -1.6798 \\
0.3 & -1.0141 & -1.859 & -2.4799 & -2.1096 & -1.9511 & -1.8591 \\
0.4 & -1.0897 & -1.997 & -2.6648 & -2.2669 & -2.0966 & -1.9977 \\
\hline
\end{tabular}

Table 4 Rate of heat transfer.

\begin{tabular}{ccccccc}
\hline \multicolumn{7}{c}{$-\left(\boldsymbol{R}_{\boldsymbol{a}_{\zeta}}\right)^{-\frac{\mathbf{1}}{4}} \boldsymbol{G}^{\prime}(\mathbf{0})=\mathbf{N} \boldsymbol{u}_{\xi}$} \\
\hline $\boldsymbol{R}_{\boldsymbol{a} \zeta}$ & $\boldsymbol{M}=\mathbf{0 . 0}$ & $\boldsymbol{M}=\mathbf{2 . 0}$ & $\boldsymbol{M}=\mathbf{4 . 0}$ & $\boldsymbol{Q}=\mathbf{0 . 0}$ & $\boldsymbol{Q}=\mathbf{0 . 2}$ & $\boldsymbol{Q}=\mathbf{0 . 5}$ \\
\hline 0.1 & 0.1616 & 0.12771 & 0.10307 & 0.4239 & 0.1416 & 0.12771 \\
0.2 & 0.1359 & 0.10739 & 0.08667 & 0.3565 & 0.1192 & 0.10739 \\
0.3 & 0.1228 & 0.09704 & 0.07832 & 0.3221 & 0.1076 & 0.09704 \\
0.4 & 0.1142 & 0.09031 & 0.07288 & 0.2997 & 0.1001 & 0.09031 \\
\hline
\end{tabular}


http://wjst.wu.ac.th

Table 5 Rate of mass transfer.

\begin{tabular}{ccccccc}
\hline \multicolumn{7}{c}{$-\left(\boldsymbol{R}_{\boldsymbol{a}_{\zeta}}\right)^{-\frac{1}{4}} \boldsymbol{J}^{\prime}(\mathbf{0})=\boldsymbol{S h}_{\xi}$} \\
\hline $\boldsymbol{R}_{\boldsymbol{a}_{\zeta}}$ & $\boldsymbol{M}=\mathbf{0 . 0}$ & $\boldsymbol{M}=\mathbf{2 . 0}$ & $\boldsymbol{M}=\mathbf{4 . 0}$ & $\boldsymbol{Q}=\mathbf{0 . 0}$ & $\boldsymbol{Q}=\mathbf{0 . 2}$ & $\boldsymbol{Q}=\mathbf{0 . 5}$ \\
\hline 0.1 & 11.136 & 11.4992 & 11.7639 & 0.5758 & 6.60381 & 11.4992 \\
0.2 & 9.3644 & 9.66967 & 9.89227 & 0.4842 & 5.55312 & 9.66967 \\
0.3 & 8.4616 & 8.73753 & 8.93868 & 0.4375 & 5.01781 & 8.73753 \\
0.4 & 7.8745 & 8.13119 & 8.31838 & 0.4072 & 4.66960 & 8.13119 \\
\hline
\end{tabular}

\section{Conclusions}

The projected observations of the numerically assessed 2D natural convective MHD flow of nanofluid with Brownian motion, heat source and other pertinent parameters over a vertical cone is itemized here. The conclusions have been reached for diverse values of the physical variables, and the significant outcomes are:

- The nano-fluid is a key factor for heat transfer enhancement, and the highest values in percentage of heat transfer enhancement may be obtained when applying exothermic $\left(K_{r}<0\right)$ chemical reactions.

- The effect of Hartmann number/heat source is more pronounced at low Rayleigh number than at high Rayleigh number because of conduction domination at low Rayleigh number.

- $Q<0$ and $K_{r}<0$ play significant roles in the present research. During exothermic chemical reaction $\left(K_{r}<0\right)$, the molar concentration increases due to release of the thermal energy from the surface of the cone to the fluid medium. Also, $\operatorname{sink}(Q<0)$ indicates that heat is diffused from the free stream to the surface of the cone and, so, the molecules absorb energy in the form of heat, which implies the free movement of molecules towards free stream.

- As $\mathfrak{R}_{a_{\zeta}}$ increases from $\frac{1}{10}$ up to $\frac{4}{10}$, the role of convection in heat transfer becomes more important and, consequently, the thermal boundary layer on the surface of the cone becomes thinner.

- Maximum value of enhancement in $F^{\prime}$ is obtained at $N_{b}=4.0$ for $K_{r}=-0.2$, whereas the minimum value of heat transfer enhancement is obtained at $N_{b}=1.0$ for $K_{r}=0.2$. Similar behavior occurs in $F^{\prime}$ for heat source and sink.

- There is a rise in the thickness of momentum boundary layer and thermal boundary layer that can be seen with the upsurge values of $N_{b}$ and $D u$.

- Higher augmentation in $J$ is calculated at $N_{t}=0.4$ for $K_{r}=-0.2$, while the least value of mass transfer enhancement is achieved at $N_{t}=0.1$ for $K_{r}=0.2$. The phenomena of thermophoretic force or diffusion thermo can be seen in the application of complex formation of protein and microfluidic devices and many others.

- Enlarged values of Brownian motion parameter lead to enhancement of the temperature, while the concentration drops.

- Application of Dufour number with MHD can be experienced in areas such as hydrology, petrology, and geosciences. 
http://wjst.wu.ac.th

\section{References}

[1] SUS Choi and JA Eastman. Enhancing thermal conductivity of fluids with nanoparticles. In: Proceedings of the ASME International Mechanical Engineering Congress and Exposition, San Francisco, CA, USA. 1995, p. 99-105.

[2] V Buddakkagari and M Kumar. transient boundary layer laminar free convective flow of a nanofluid over a vertical cone/plate. Int. J. Appl. Comput. Math. 2015; 1, 427-48.

[3] CY Cheng. Natural convection boundary layer flow over a truncated cone in a porous medium saturated by a nano-fluid. Int. Comm. Heat Mass Tran. 2012; 39, 231-5.

[4] RSR Gorla, AJ Chamkha and V Ghodeswar. Natural convective boundary layer flow over a vertical cone embedded in a porous medium saturated with a nano-fluid. J. Nanofluid. 2014; 3, 65-71.

[5] A Behseresht, A Noghrehabadi and M Ghalambaz. Natural-convection heat and mass transfer from a vertical cone in porous media filled with nano-fluids using the practical ranges of nano-fluids thermophysical properties. Chem. Eng. Res. Des. 2014; 92, 447-52.

[6] A Mahdy. Natural convection boundary layer flow due to gyrotactic microorganisms about a vertical cone in porous media saturated by a nano-fluid. J. Braz. Soc. Mech. Sci. Eng. 2016; 38, $67-$ 76.

[7] EA Sameh and A Mahdy. Natural convection flow and heat transfer enhancement of a nano-fluid past a truncated cone with magnetic field effect. World J. Mech. 2012; 2, 272-9.

[8] AJ Chamkha, S Abbasbandy and AM Rashad. Non-darcy natural convection flow of non-newtonian nano-fluid over a cone saturated in a porous medium with uniform heat and volume fraction fluxes, Int. J. Numer. Meth. Heat Fluid Flow. 2015; 25, 422-37.

[9] D Kalita, S Hazarika and S Ahmed. Applications of CNTs in a vertical channel of porous medium for human blood flow: A rheological model. JP J. Heat Mass Tran. 2020; 20, 105-20.

[10] CSK Raju, N Sandeep and A Malvandi. Free convective heat and mass transfer of MHD nonnewtonian nano-fluids over a cone in the presence of non-uniform heat source/sink. J. Mol. Liq. 2016; 221, 108-15.

[11] M Sheikholeslami, MM Rashidi, T Hayat and DD Ganji. Free convection of magnetic nano-fluid considering MHD viscosity effect. J. Mol. Liq. 2016; 218, 393-9.

[12] AJ Chamkha, S Abbasbandy, AM Rashad and K Vajravelu. Radiation effects on mixed convection about a cone embedded in a porous medium filled with a nano-fluid. Meccanica 2013; 48, 275-85.

[13] S Siddiqa, N Begum and MA Hossain. Radiation effects from an isothermal vertical wavy cone with variable fluid properties. Appl. Math. Comput. 2016; 289, 149-58.

[14] R Kandasamy, Muhaimin, I Hashim and Ruhaila. Thermophoresis and chemical reaction effects on non-darcy mixed convective heat and mass transfer past a porous wedge with variable viscosity in the presence of suction or injection. Nucl. Eng. Des. 2008; 238, 2699-705.

[15] AM Rashad, MA El-Hakiem and MMM Abdou. Natural convection boundary layer of a nonnewtonian fluid about a permeable vertical cone embedded in a porous medium saturated with a nano-fluid. Comput. Math. Appl. 2011; 62, 3140-51.

[16] PS Reddy and AJ Chamkha. Heat and mass transfer analysis in natural convection flow of nanofluid over a vertical cone with chemical reaction. Int. J. Numer. Meth. Heat Fluid Flow 2017; 27, 222.

[17] D Kalita, S Hazarika and S Ahmed. MHD drag force on water based cylindrical shaped Zno nanoparticle in a chemically reacting nano-fluid through channel: A theoretical investigation. Ann. Facul. Eng. Hunedoara Int. J. Eng. 2020; 18, 23-32.

[18] M Mustafa, JA Khan, T Hayat and A Alsaedi. Buoyancy effects on the MHD. Int. J. Heat Mass Tran. 2017; 108, 1340-6.

[19] FO Patrulescu, T Grosan and I Pop. Mixed convection boundary layer flow from a vertical truncated in a nano-fluid. Int. J. Numer. Meth. Heat Fluid Flow 2014; 24, 1175-90.

[20] AM Rashad, B Mallikarjuna, AJ Chamkha and SH Raju. Thermophoresis effect on heat and mass transfer from a rotating cone in a porous medium with thermal radiation. Afrika Matematika 2016; 27, 1409-24. 
http://wjst.wu.ac.th

[21] H Rosali, A Ishak, R Nazar and I Pop. Mixed convection boundary layer flow past a vertical cone embedded in a porous medium subjected to a convective boundary condition. Propul. Power Res. 2016; 5, 118-22.

[22] S Saleem, S Nadeem and RUI Haq. Buoyancy and metallic particle effects on an unsteady waterbased fluid flow along a vertically rotating cone. Eur. Phys. J. Plus. 2014; 129, 213.

[23] S Hazarika, S Ahmed and AJ Chamkha. Investigation of nanoparticles $\mathrm{Cu}, \mathrm{Ag}$ and $\mathrm{Fe}_{3} \mathrm{O}_{4}$ on thermophoresis and viscous dissipation of MHD nanofluid over a stretching sheet in a porous regime: A numerical modelling. Math. Comput. Simulat. 2021; 182, 819-37.

[24] HL Dufour. Ueber die Diffusion der Gase durch poröse Wände und die sie begleitenden Temperaturveränderungen. Ann. Phys. 1873; 148, 490.

[25] H Darcy. Les fontaines publiques de la ville de dijon. Dalmont, Paris, 1856.

[26] R Brown. A brief account of microscopical observations made in the months of June, July and August, 1827, on the particles contained in the pollen of plants; and on the general existence of active molecules in organic and inorganic bodies. Phil. Mag. 1828; 4, 161-73.

[27] J Buongiorno. Convective transport in nanofluids. J. Heat Tran. 2006; 128, 240-50.

[28] M Sheikholeslami, M Jafaryar, Z Said, AI Alsabery, H Babazadeh and A Shafee. Modification for helical turbulator to augment heat transfer behavior of nanomaterial via numerical approach. Appl. Therm. Eng. 2021; 182, 115935.

[29] M Sheikholeslami, SA Farshad, Z Ebrahimpour and Z Said. Recent progress on flat plate solar collectors and photovoltaic systems in the presence of nanofluid: A review. J. Cleaner Prod. 2021; 293, 126119.

[30] M Sheikholeslami, SA Farshad and Z Said. Analyzing entropy and thermal behavior of nanomaterial through solar collector involving new tapes. Int. Commun. Heat Mass Tran. 2021; 123, 105190.

[31] M Sheikholeslami and SA Farshad. Investigation of solar collector system with turbulator considering hybrid nanoparticles. Renew. Energ. 2021; 171, 1128-58.

[32] S Hazarika and S Ahmed. Theoretical investigation of viscosity and thermal conductivity of a gas along a non-isothermal vertical surface in porous environment with dissipative heat: Numerical technique. J. Appl. Comput. Mech. 2021. https://dx.doi.org/10.22055/jacm.2021.35353.2638. 